\title{
Saúde emocional e sentimentos de mães de bebês em UTIN
}

\author{
Carolina Daniel Montagner* \\ Olga Maria Piazentin Rolim Rodrigues** \\ Maithê Christine Prampero*** \\ Nadja Guazzi Arenales**** \\ Nathália Maria Capellini******
}

\begin{abstract}
Resumo
Pretendeu-se descrever a saúde emocional (indicadores de estresse, depressão e ansiedade) e os sentimentos de 109 mães em diferentes momentos da internação do seu bebê em Unidade de Terapia Intensiva Neonatal (UTIN) (por ocasião da notícia da internação e nas visitas). Elas responderam ao Protocolo de identificação de sentimentos maternos relativos à internação de bebês e aos instrumentos para avaliação da saúde emocional (estresse, ansiedade e depressão). As mães relataram mais sentimentos negativos por ocasião da internação $(86,2 \%)$ e mais sentimentos positivos durante as visitas $(60,6 \%)$. Quanto à saúde emocional, 25\% das mães relataram sintomas clínicos para ansiedade, depressão e estresse. Ansiedade Estado foi mais frequente entre as mães que relataram sentimentos negativos na internação, enquanto a depressão o foi entre as mães que relataram sentimentos positivos nas visitas. Observou-se que as mães se tornaram mais otimista no decorrer da internação do bebê, todavia, necessitam de apoio terapêutico nesta hora. Palavras-chave: sentimentos maternos, saúde emocional materna, internação, UTIN, bebês.
\end{abstract}

\begin{abstract}
It was intended to describe the emotional health (indicators of stress, depression and anxiety) and the feelings of 109 mothers at different times of their baby's hospitalization in the Neonatal Intensive Care Unit (NICU) (at the time of the hospitalization news and during visits). They responded to the Protocol for the identification of maternal feelings regarding the hospitalization of babies and the instruments for assessing emotional health (stress, anxiety and depression). Mothers reported more negative feelings during hospitalization $(86.2 \%)$ and more positive feelings during visits $(60.6 \%)$. As for emotional health, $25 \%$ of mothers reported clinical symptoms for anxiety, depression and stress. State anxiety was more frequent among mothers who reported negative feelings during hospitalization, while depression was more frequent among mothers who reported positive feelings during visits. It was observed that mothers became more optimistic during the baby's hospitalization, however, they need therapeutic support at this time. Keywords: maternal feelings, maternal emotional health, hospitalization, NICU, babies.
\end{abstract}

* ORCID iD https://orcid.org/0000-0002-4533-7286. URL https://orcid.org/0000-0002-4533-7286. Universidade Estadual Paulista "Júlio de Mesquita Filho" - Unesp. Doutoranda no Programa de Pós-graduação em Psicologia do Desenvolvimento e Aprendizagem, Faculdade de Ciências, Unesp/Bauru. E-mail: carol.montanhaur@gmail.com.

** ORCID iD https://orcid.org/0000-0002-5332-256X. URL https://orcid.org/0000-0002-5332-256X. Universidade Estadual Paulista "Júlio de Mesquita Filho" - Unesp. Professora Doutora em Psicologia do Desenvolvimento e Aprendizagem, Faculdade de Ciências, Unesp/Bauru. E-mail: olgarolim29@ gmail.com.

*** ORCID iD https://orcid.org/0000-0001-8798-8952. URL https://orcid.org/0000-0001-8798-8952. Pontifícia Universidade Católica de Campinas - PUCCAMP. Psicóloga residente no Programa de Residência Multiprofissional em Saúde da Mulher, da Pontifícia Universidade Católica - PUC de Campinas (PUCCAMP). E-mail: maitheprampero@gmail.com .

**** ORCID iD https://orcid.org/0000-0001-6514-1726. URL https://orcid.org/0000-0001-6514-1726. Consultório particular. Médica e mestre em Biotecnologia pela Unesp/Botucatu. E-mail: nadja_arenales@yahoo.com.br .

***** ORCID iD https://orcid.org/0000-0002-8720-5812. URL https://orcid.org/0000-0002-8720-5812. Médica em posto de saúde. Médica pela Pontifícia Universidade Católica - PUC de Campinas (PUCCAMP). E-mail: nathcapellini@hotmail.com . 


\section{Introdução}

A Organização Mundial de Saúde (2018) destacou que, a cada ano, mais de trinta milhões de recém-nascidos necessitaram de internação em UTIN, por motivos que incluem malformações congênitas, lesões cerebrais, complicações da prematuridade ou icterícia. Estes dados reforçam a importância da assistência ao recém-nascido, com estrutura adequada e equipe especializada, que coloque o bebê como o centro dos cuidados, sem negligenciar sua família.

A internação introduz o bebê, logo em suas primeiras horas de vida, em um ambiente repleto de estímulos como barulho, luz, movimentação de profissionais, intervenções invasivas e constantes como aspiração, intubação, cateterismo, punção, dentre outros, ao longo de todo período de internação. São fatores que podem atuar como estressores ao bebê e, por consequência, para a mãe que o visita (Jordão, Pinto, Machado, Costa \& Trajano, 2016). Além do ambiente hostil, o início da internação é um momento de dificuldade para os familiares, pois há uma separação abrupta logo após o nascimento resultando em pouco contato físico e visual entre a díade. Enquanto internado esse bebê pode, muitas vezes, responder pouco ou não responder às tentativas de interação dos pais. Além disso, eles dividem a atenção e os cuidados com os profissionais da unidade, podendo se sentir sem ter o controle da situação e dos cuidados com o próprio filho (Neves, Zimmermann \& Boering, 2021; Veronez, Borghesan, Corrêa \& Higarashi, 2017).

Ver constantemente seu bebê preso a fios, equipamentos e, quase sempre dormindo, tende a produzir na mãe insegurança com relação à sua sobrevida fora daquele ambiente (Neves et al., 2021). Para Coelho, Custódio, Rosso, Silva, Silva e Carniel 2018) é fundamental que ocorra acolhimento e aceitação do cuidado humanizado por parte dos profissionais de saúde, mas também, que sejam promovidos espaços de acolhimento focados nos pais e mães dos bebês. O cuidado humanizado se opõe a um cuidado mecânico, mais tecnicista. Ele implica na promoção de bem-estar e qualidade de vida ao indivíduo internado e, também, a associação com a família, estimulando a interação com o bebê, por meio da amamentação, por exemplo (Roseiro \& Paula, 2015). É importante que os profissionais da equipe de saúde orientem os pais antes da primeira visita, descrevendo a rotina e apresentando os equipamentos da UTIN, de modo a não amedrontar os pais (Fonseca, 2016). A inclusão dos pais nos cuida- dos e a promoção de informações sobre a convivência com o bebê pós-internação também são formas de propiciar comunicação entre profissionais da saúde e pais, constituindo possibilidades de cuidados humanizados para o bebê e seus cuidadores (Coelho et al., 2018).

O momento de internação do bebê pode vir ou não de maneira inesperada. Há casos em que a gestação corre riscos e um parto prematuro é necessário e, ás vezes previsível, mas, há casos em que a gestação corre bem, mas o bebê nasce com alguma condição que exige internação para atenção intensiva, configurando situações que podem gerar angústia e sentimentos de confronto, além de impedir um primeiro contato entre a díade mãe-bebê (Fonseca, 2016). O momento de separação entre a mãe e o bebê que necessita de cuidados intensivos, é complexo e gera emoções e sentimentos maternos diversos, além de motivar dúvidas, medos e incertezas que advém do risco que o bebê corre, há sentimentos positivos relacionados com o nascimento e sobrevida do bebê (Zani \& Alvim, 2017).

Identificar os sentimentos e experiências das primeiras visitas e contatos dos pais com os bebês internados foram os objetivos de diversos pesquisadores (Cordeiro, Cavalcante, Duarte, Figueira, Jacob \& Pimenta, 2018; Hagen, Iversen \& Svindseth, 2016; Melo, Araújo, Bezerra, Santos, Marques \& Fernandes, 2016; Neves et al., 2021; Pinto, Padovani \& Linhares, 2009; Zani \& Alvim, 2017). Pinto et al. (2009) realizaram um estudo analisando as expectativas, concepções e reações em relação ao bebê e à internação. Os resultados demonstraram que, no geral, as mães tinham expectativas positivas em relação ao bebê (saudável, desejo alta hospitalar e bom desenvolvimento posterior, boa evolução do caso), concepções positivas também (amor e orgulho do bebê) e, também, reações negativas frente ao bebê e à internação (choro, tristeza, raiva, medo, angústia, insegurança, revolta).

No estudo de Zani e Alvim (2017) sobre a maternagem hospitalizada e os sentimentos decorrentes da internação do filho prematuro e baixo peso buscou avaliar os sentimentos advindos dessa experiência materna e pode-se perceber sentimentos de sofrimento relacionados ao inesperado, felicidade diante do nascimento, tristeza na internação, dificuldade de aceitação da separação do filho, apoio da família nas visitas ao bebê e confiança na equipe. Observa-se ambivalência de sentimentos positivos e negativos na vivência de maternidade.

No estudo de Melo et al. (2016) foram entrevistadas mães de bebês hospitalizados sobre seus sentimentos 
diante desse processo. Os autores perceberam a preocupação em relação aos cuidados prestados pela equipe e compreensão da necessidade desses cuidados específicos para a boa recuperação do bebê, mas também, ressaltaram a necessidade de atenção da equipe de saúde para os sentimentos das mães e para a sua participação em algumas tarefas de cuidado ao bebê, para favorecer a vinculação da díade.

Cordeiro et al. (2018) encontraram duas temáticas recorrentes nas falas de mães de bebês em UTIN: as experiências que vivenciaram na unidade e a confiança na assistência prestada pelos profissionais. Sobre as vivências, as mães relataram sentimentos de medo nos momentos que se deparavam com a separação ao filho, pois tinham incerteza sobre a cura ou morte do bebê e, em menor porcentagem, sentimentos positivos relacionados a fé e esperança. Em relação a segunda temática, as mães relataram confiança diante dos cuidados, tendo como pontos positivos a disponibilidade dos profissionais para informar sobre a condição de saúde do bebê e o ambiente acolhedor.

As sete mães que participaram do estudo de Fonseca (2016) relataram desamparo, medo e angústia, além de frustração pela ausência do bebê em casa, no quarto que o espera e, também, frustração por não pegar o bebê. Entre os sentimentos relatados pelas mães estavam: susto, vulnerabilidade, medo, confusão, insegurança, preocupação, dor, ansiedade e culpa. Ao mesmo tempo as mães relataram o papel da maternidade como algo único, sentimento incrível e a realização de um sonho.

A ambivalência de sentimentos também foi observada no estudo de Hagen et al. (2016), realizado com pais e mães de bebês internados. Os autores encontraram sentimentos contraditórios como alegria, tristeza, esperança e desespero.

Com o objetivo de analisar a percepção de mães sobre o processo de internação de seus filhos em UTIN, Neves et al. (2021) entrevistaram 10 mães de bebês. Os resultados apontaram para sentimentos ambivalentes motivados pela insegurança e medo, considerando as intercorrências clínicas relacionadas à saúde de seus bebês. Os dados obtidos sugeriram a necessidade de apoio terapêutico a essas mães.

Em estudo de revisão de pesquisas sobre a identificação dos sentimentos envolvidos na internação de bebês, Silva, Menezes, Cardoso e França (2016) apontaram para a recorrência de sentimentos ambíguos. Os primeiros momentos, como a notícia da internação, as mães relataram mais sentimentos negativos, relacionados a tristeza, angústia, medo da sobrevida do bebê, associação entre o ambiente hospitalar (UTIN) com a morte eminente, culpa recorrente. Mas, após alguns dias de convivência com o ambiente, relataram sentimentos de esperança e felicidade.

A saúde emocional da mãe que tem bebê internado em UTIN também merecem atenção, o que reforça a importância de avaliar a presença de indicadores de ansiedade, depressão e estresse, diretamente atrelados à condição de internação do bebê, à rede de apoio e/ou à características da personalidade da mãe. Ao serem avaliadas 50 mães de bebês internados em UTIN apresentaram prevalência de indicadores clínicos para ansiedade, depressão e estresse na população, encontrando participantes que apresentaram mais de um indicador clínico, tendo resultados mais preocupantes as mães que permaneciam mais tempo com seus bebês internados (Montanhaur, Rodrigues \& Arenales, 2021).

Correia, Carvalho e Linhares (2008) comparando as verbalizações entre mães com ou sem indicadores clínicos de saúde emocional encontraram verbalizações negativas em ambos os grupos, sendo mais recorrentes em mães que apresentaram sintomas clínicos para ansiedade e depressão, relatando medo, receio de aproximação do filho, revolta e negação. Além disso, no grupo de mães com indicadores emocionais, o tempo de permanência desse bebê hospitalizado foi maior, podendo ser um fator importante para a percepção negativa e prejuízo para a saúde emocional.

Resultados semelhantes foram encontrados no estudo de Pinto et al. (2009) que apontaram que as mães com indicadores clínicos demonstraram mais reações negativas, quando comparadas às mães sem indicadores clínicos. Ainda, as concepções maternas positivas relacionaram-se diretamente à maior idade gestacional, menor tempo de internação e menor ansiedade-estado da mãe.

Além da depressão, a ansiedade pode, também, surgir em mães de bebês hospitalizados. Cruz Dantas, Araújo, Revorêdo, Pereira e Maia (2015) compararam a saúde emocional de mães de bebês internados em UTIN com mães de bebês não internados e comprovaram que as mães de bebês internados apresentaram significativamente mais indicadores de Ansiedade Traço que as mães do grupo controle, sendo a hospitalização um fator de risco para a saúde mental materna. 
Margaret, Lewis, Bhat, Nayak, Pai e Mundkur(2018) realizaram um estudo com mães de bebês internados em UTIN com o objetivo de correlacionar a depressão pós-parto com a vinculação mãe-bebê. De 100 participantes, $25 \%$ apresentaram sintomas para depressão moderada e $17 \%$ para depressão severa. Os achados mostraram uma correlação negativa entre depressão pós-parto e vínculo diádico, o que representa que maiores índices de depressão representam maior comprometimento ao vínculo. Estes dados reforçam a necessidade de acolhimento para as mães de bebês internados, bem como avaliações de saúde emocional materna e suporte psicológico, a fim de auxiliar estas mulheres em sofrimento e promover uma interação saudável entre mães e bebês.

O estresse é outra categoria que diz respeito à saúde emocional materna e que pode estar presente nos momentos de internação do bebê. Fatores ambientais, como os aparelhos que envolvem o bebê, a falta de informações que muitas vezes permeia a relação com a equipe de saúde e a expectativa da alta, foram objeto do estudo de Veronez et al. (2017). Os autores apresentaram relatos de mães que caracterizaram a espera pela alta como um momento de estresse, que deixa "os nervos à flor da pele", como relatado por uma das sete participantes. Segundo os autores, a expectativa da alta deixa as mães ansiosas e, também, estressadas, pois como é difícil prever uma data específica em que o bebê receberá alta, a espera é constante. Enquanto isso, outros filhos demandam atividades e presença da mãe, além de conflitos gerados com companheiros, que também são fatores que compõem a multideterminação do estresse neste momento.

Investigar a saúde emocional materna e os sentimentos decorrentes da hospitalização de bebês possibilitaria o maior conhecimento dessa população, das alterações que perpassam esse período para, em seguida, idealizar planos de intervenções individuais ou grupais que auxiliem as mães a terem uma vivência razoável desse período e possam fazer uma tranquila transição para a parentalidade.

O presente estudo pretendeu: a) descrever os sentimentos das mães em diferentes momentos da internação (por ocasião da notícia da internação e da visita à UTI) e indicadores da sua saúde emocional (de estresse, depressão e ansiedade); b) comparar os indicadores clínicos emocionais de grupos: 1) que permanecem com sentimentos negativos nos dois momentos; 2) que permanecem com sentimentos positivos nos dois momentos e, 3) que mudam de sentimentos negativos no primeiro momento, para positivos no segundo momento.

\section{Método}

Esta pesquisa é parte integrante do projeto "XXXXXX" aprovado pelo Comitê de Ética da Faculdade XXXX (Processo n ${ }^{\circ}$ XXXX).

\section{Participantes}

Participaram da pesquisa 109 mães de bebês internados na Unidade de Terapia Intensiva Neonatal (UTIN) da Maternidade XXXXXXX, da cidade de XXXXXXXX, que atende, prioritariamente, usuárias do SUS. Dessas mães 65,2\% (71 mães) tinham até 30 anos (Min= 14, Max $=42$, Média $=27, D P=7,2)$. Em relação a escolaridade $66,1 \%$ delas (72 participantes) haviam completado o ensino médio, que corresponde aproximadamente a nove anos de estudo (Min= 4 anos, $\mathrm{Max}=16$, Média $=$ $9,7, \mathrm{DP}=2,2)$. A maioria das mães, somando $67,9 \%$ (74 participantes) tinham de um a dois filhos ( $\mathrm{Min}=1, \mathrm{Max}=$ 6, Média $=2,1$ e DP= 1,2). Também houve maior número de mães, 54,1\% (59 participantes) que se encontravam em união estável, que não haviam planejado a gestação $(62,4 \%)$, que haviam passado por parto cesariano $(57,8 \%)$ e, de acordo com o Critério de Classificação Econômica Brasil da Associação Brasileira de Empresas de Pesquisas (ABEP), pertenciam a classe econômica $\mathrm{C}(71,6 \%)$.

Dos bebês das mães avaliadas $57,3 \%$ eram meninas (63 participantes) e tinham em média 32,1 semanas de idade gestacional, sendo o mínimo de 24 semanas e o máximo de 40 semanas $(\mathrm{DP}=4,4)$ tendo em sua maioria $(65,4 \%)$ nascidos de até 33 semanas de gestação (72 participantes). Os neonatos pesavam em média 1856 gramas, sendo o mínimo de 550 gramas e o máximo de 4196 gramas $(\mathrm{DP}=943,5)$ e 66,3\% deles (84 participantes) pesavam até 2500 gramas. No momento da entrevista esses bebês estavam, em média, há 9,5 dias internados sendo o mínimo de um dia e o máximo de 40 dias (DP= $8,0)$ visto que $71,8 \%$ deles ( 79 participantes) estavam há, no máximo, 10 dias internados.

\section{Local}

Os dados foram coletados em uma sala para atendimento individual, nas dependências da Unidade de Terapia Intensiva Neonatal (UTIN), da Maternidade XX, localizada em uma cidade de XXXXXX, no centro oeste paulista. 


\section{Instrumentos}

O Questionário Sociodemográfico continha espaços para a coleta de informações sobre as mães (idade, profissão, escolaridade, número de filhos) e sobre o bebê (sexo, problema de saúde que o levou à internação).

O Critério de Classificação Econômica Brasil CCEB é uma escala organizada pela Associação Brasileira de Empresas de Pesquisa (Abep, 2013) em perguntas sobre variáveis (utensílios domésticos, quantidade de cômodos etc.) e serviços públicos (água encanada, rua pavimentada) utilizadas para indicar o nível socioeconômico do entrevistado.

O "Protocolo de identificação de sentimentos maternos na internação de bebês (PISMIB)" foi elaborado por Montanhaur (2018) com o objetivo de avaliar sentimentos vivenciados pelas mães em diferentes períodos da internação, bem como a justificativa para a emergência desses sentimentos. Consta de três afirmativas, referente a uma das situações avaliadas: no momento da notificação da necessidade de internação, das visitas ao bebê e na expectativa da alta hospitalar, seguidas por um conjunto de sentimentos positivos e negativos.

Para avaliação da saúde emocional foram utilizados os instrumentos: Inventário de Depressão de Beck II (BDI-II), Inventário de Ansiedade Traço-Estado (IDATE) e Escala de Estresse Percebido (Perceived Stress Scale - PSS). O BDI-II é composto por 21 questões, em que cada uma possui quatro alternativas ( 0 a 4 pontos). A pontuação máxima que pode ser obtida é 63 pontos e, para critério clínico, foi utilizado o valor de corte igual a 14 pontos.

O IDATE é um instrumento composto por duas partes: Primeira Parte, que avalia a Ansiedade Estado, diz respeito aos sentimentos atribuídos ao momento em que o teste é respondido e, a Segunda Parte, que avalia a Ansiedade Traço, considera sentimentos que ocorrem com frequência correspondendo a uma característica constante da personalidade do indivíduo. Cada parte do inventário é composta por vinte itens que variam de 1 a 4 pontos, segundo uma escala Likert. De modo a considerar indicadores clínicos, foram utilizadas as seguintes pontuações: 48 pontos para a Ansiedade Estado e 49 pontos para Ansiedade Traço.

O PSS contém 14 questões, com questões positivas e negativas. As questões positivas obtêm pontuação invertida na escala Likert, a qual varia de 0 a 4 pontos, em que zero corresponde a Nunca e 4 a Sempre. A pontuação de corte, a partir da qual se considera um indicador clínico, é de 30 pontos (Faro, 2015).

\section{Procedimento de coleta de dados}

Durante as visitas à UTIN as mães eram convidadas a participar da pesquisa. Em caso de aceite, em uma sala reservada, a mãe respondia aos instrumentos. Em caso de identificação de indicadores clínicos as participantes eram encaminhadas para serviços de Psicologia disponíveis na comunidade.

\section{Procedimento de Análise de dados}

Os dados sociodemográficos foram tabulados e organizados em dados sobre as mães, bebês, condição de nascimento, tempo de internação e descritos em frequência e média. Os resultados obtidos no "Protocolo de identificação de sentimentos maternos na internação de bebês (PISMIB)" foram organizados pelos momentos avaliados (diante da notícia da internação e durante as visitas) e categorizados em sentimentos positivos e negativos e descritos em frequência absoluta e relativa.

Os resultados obtidos nos instrumentos de avaliação da saúde emocional materna foram tabulados e corrigidos conforme seus manuais. Adotou-se para o instrumento IDATE o critério de percentil maior que 75 para consideração de sintomas clínicos para Ansiedade-Estado e Ansiedade-Traço, conforme utilizado por Schiavo, Rodrigues e Perosa (2014). No Inventário de Depressão de Beck - BDI-II adotou-se a classificação da pontuação de média a alta como indicador clínico para depressão. $\mathrm{Na}$ Escala de Estresse Percebido (Perceived Stress Scale - PSS), adotou-se a classificação adota por Faro (2015) para estresse em intensidade em alta e muito alta como critério para sintomas clínicos para estresse.

Os dados de sentimentos e saúde emocional foram comparados utilizando o Pacote estatístico SPSS (SPSS,) aplicando o teste Mann-Whitney para as comparações entre grupos, utilizando o valor de significância de $\mathrm{p}<0,05$.

\section{Resultados}

A partir do "Protocolo de identificação de sentimentos maternos na internação de bebês (PISMIB)" foi possível identificar os sentimentos vivenciados pelas mães em dois momentos diferentes, diante da notícia da internação e nos momentos de visitas aos bebês. Quando questionadas sobre os sentimentos que envolviam a notícia da internação $86,2 \%$ das mães (94 participantes) relataram sentimentos 
negativos como culpa, decepção, preocupação, nervosismo ou desespero, enquanto 13,8\% relataram positivos. Entretanto, em relação aos sentimentos desencadeados durante a internação $60,6 \%$ das mães (66 participantes) relataram sentimentos positivos como tranquilidade, conformismo, confiança e amparo enquanto 37,6\% delas (41 participantes) relataram sentimentos negativos (Tabela 1$)$.

Tabela 1 - Distribuição dos sentimentos positivos e negativos por ocasião da notícia e durante a internação.

\begin{tabular}{|l|l|l|l|l|l|}
\hline & \multicolumn{2}{|l|}{$\begin{array}{l}\text { Notícia de } \\
\text { internação }\end{array}$} & \multicolumn{2}{|l|}{$\begin{array}{l}\text { Durante a } \\
\text { internação }\end{array}$} \\
\hline Sentimentos & FA & FR & FA & FR \\
\hline $\begin{array}{l}\text { Positivos (tranquila, conformada, } \\
\text { confiante, amparada) }\end{array}$ & 15 & 13,8 & 66 & 60,6 \\
\hline $\begin{array}{l}\text { Negativos (culpada, decepcionada, } \\
\text { preocupada, nervosa, desesperada) }\end{array}$ & 94 & 86,2 & 41 & 37,6 \\
\hline Não responderam & 0 & 0 & 02 & 1,8 \\
\hline & 109 & 100 & 109 & 100 \\
\hline
\end{tabular}

Fonte: dados da pesquisa

Para identificação das características da saúde emocional das mães da amostra foram aplicados os instrumentos IDATE, BDI e PSS para avaliação de sintomas de ansiedade traço e estado, depressão e a presença de estressores, cujos dados estão apresentados na Tabela 2. Em termos de Ansiedade, 51,4\% (56 participantes) das mães apresentaram pontuação clínica para a Ansiedade Estado e 36,7\% (40 participantes) apresentaram pontuação considerada clínica para Ansiedade Traço. Dos dados obtidos na Escala de Estresse Percebido (Perceived Stress Scale - PSS), foi possível notar que $24,8 \%$ delas (27 participantes) apresentaram pontuação clínica e, em relação à Depressão, 24,8\% (27 participantes) obtiveram pontuação considerada clínica no Inventário de Depressão de Beck (BDI-II).
Tabela 2 - Descrição dos indicadores emocionais clínicos e não clínicos.

\begin{tabular}{|l|l|l|l|l|l|}
\hline & Clínico & & $\begin{array}{l}\text { Não- } \\
\text { clínico }\end{array}$ & & Total \\
\hline & FA & FR(\%) & FA & FR(\%) & \\
\hline $\begin{array}{l}\text { Ansiedade } \\
\text { Estado }\end{array}$ & 56 & 51,4 & 53 & 48,6 & 109 \\
\hline Ansiedade Traço & 40 & 36,7 & 69 & 63,3 & 109 \\
\hline Estresse & 27 & 24,8 & 82 & 75,2 & 109 \\
\hline Depressão & 27 & 24,8 & 82 & 75,2 & 109 \\
\hline
\end{tabular}

Fonte: dados da pesquisa

Considerando o número de indicadores clínicos observou-se que 33,9\% (37) das participantes não apresentaram indicadores clínicos para Ansiedade Traço-Estado, Estresse ou Depressão. Delas, 44,9\% (49) apresentaram de 1 a 2 indicadores emocionais clínicos e 21,1\% (23) apresentaram 3 a 4 indicadores clínicos das categorias analisadas.

Observando os sentimentos positivos e negativos relatados pelas mães no momento da notícia e da visita ao bebê na unidade neonatal e o número de indicadores clínicos apresentados por elas foi possível notar que, no momento da notícia houve prevalência de sentimentos negativos e, das participantes nesta condição 59,5\% apresentaram pelo menos um indicador clínico de saúde emocional e, entre as que relataram sentimentos positivos 6,3\% também apresentaram pelo menos um indicador clínico. Em relação aos sentimentos vivenciados durante as visitas ao bebê houve prevalência dos sentimentos positivos e, das mães nessa condição $35,8 \%$ delas $(38$ participantes) apresentaram pelo menos um indicador clínico, enquanto que, das mães que relataram sentimentos negativos $27,6 \%$ também apresentaram pelo menos um indicador clínico para saúde emocional (Tabela 3).

Tabela 3 - Distribuição dos sentimentos positivos e negativos considerando o número de indicadores.

\begin{tabular}{lcccccccc}
\hline Indicadores & \multicolumn{7}{c}{ Condição } \\
\hline \multicolumn{4}{c}{$\begin{array}{c}\text { Sentimentos Posi- } \\
\text { tivos }\end{array}$} & $\begin{array}{c}\text { Sentimentos Nega- } \\
\text { tivos }\end{array}$ & \multicolumn{2}{c}{ Sentimentos Positivos } & Sentimentos Negativos \\
\hline Nenhum & $\mathrm{N}$ & $\%$ & $\mathrm{~N}$ & $\%$ & $\mathrm{n}$ & $\%$ & $\mathrm{n}$ & $\%$ \\
1 & 8 & 7,3 & 29 & 26,6 & 26 & 23,8 & 11 & 10,1 \\
2 & 1 & 0,9 & 23 & 21,1 & 12 & 11,0 & 11 & 10,1 \\
3 & 1 & 0,9 & 24 & 22,0 & 15 & 13,8 & 10 & 9,2 \\
4 & 3 & 2,7 & 9 & 8,2 & 7 & 6,4 & 4 & 3,7 \\
\hline & 2 & 1,8 & 9 & 8,2 & 5 & 4,6 & 5 & 4,6 \\
\hline
\end{tabular}


Para efeito de comparação da saúde emocional das mães diante da notícia da internação a amostra foi dividida em dois grupos, o grupo em que as mães relataram sentimentos positivos (G1) e o grupo em que elas relataram sentimentos negativos sobre a notícia (G2). Os resultados apontaram para diferença estatisticamente significativa $(\mathrm{p}=$ 0,037) entre o G1 e G2 nos escores para Ansiedade-Estado, obtendo média maior no G2 em que as mães relataram sentimentos negativos (Tabela 4).

Tabela 4 - Diferença da saúde emocional das mães que relataram sentimentos positivos e negativos diante da notícia da internação.

\begin{tabular}{|c|c|c|c|c|c|}
\hline \multirow[t]{2}{*}{$\begin{array}{l}\text { Condição } \\
\text { nal }\end{array}$} & \multicolumn{2}{|c|}{$\begin{array}{l}\text { G1 (sentimentos } \\
\text { tivos) }\end{array}$} & \multicolumn{2}{|c|}{$\begin{array}{l}\text { G2 (sentimentos } \\
\text { gativos) }\end{array}$} & \multirow[t]{2}{*}{$\mathrm{p}$} \\
\hline & Média & $\mathrm{DP}$ & Média & $\mathrm{DP}$ & \\
\hline Ansiedade-Estado & 43,3 & 9,46 & 48,5 & 8,76 & $0,037 *$ \\
\hline Ansiedade-Traço & 40,2 & 8,64 & 42,4 & 8,84 & 0,359 \\
\hline Estresse & 25,8 & 10,02 & 22,36 & 9,25 & 0,189 \\
\hline Depressão & 7,06 & 6,09 & 8,35 & 5,75 & 0,428 \\
\hline $\begin{array}{l}\text { Número de indi- } \\
\text { cadores }\end{array}$ & 1,33 & 1,63 & 1,42 & 1,28 & 0,804 \\
\hline
\end{tabular}

Fonte: dados da pesquisa $\quad$ Legenda: $\mathrm{p}<0,05^{*}$

As mães também foram divididas em dois grupos de acordo com os sentimentos relatados por elas durante as visitas, sendo G1 para sentimentos positivos e G2 para sentimentos negativos. Esses grupos foram comparados estatisticamente e observou diferença estaticamente significativa entre os grupos nos escores para depressão $(\mathrm{p}=$ 0,017), sendo a média do G2 maior que a do G1 (Tabela 5).

Tabela 5 - Diferença da saúde emocional das mães que relataram sentimentos positivos e negativos durante as visitas.

\begin{tabular}{llllll}
\hline Condição emocional & \multicolumn{4}{l}{ G1 (sentimentos positivos) } & \multicolumn{3}{l}{ G2 (sentimentos negativos) } & $\mathrm{p}$ \\
\hline Ansiedade-Estado & Média & DP & Média & DP & \\
Ansiedade-Traço & 47,1 & 9,17 & 49,0 & 8,7 & 0,292 \\
Estresse & 42,5 & 9,27 & 41,4 & 8,16 & 0,544 \\
Depressão & 22,4 & 8,72 & 22,7 & 10,13 & 0,894 \\
Número indicadores & 7,1 & 5,35 & 9,8 & 6,1 & $\mathbf{0 , 0 1 7}$ \\
\hline
\end{tabular}

Fonte: dados da pesquisa Legenda: $\mathrm{p}<0,05^{*}$

A amostra também foi dividida de acordo com os sentimentos vividos pelas mães nos dois momentos avaliados, diante da notícia da internação e das visitas ao bebê a UTIN, formando os grupos de acordo com a manutenção dos mesmos sentimentos nos dois momentos ou pela alteração dos sentimentos relatados. O G1 foi formado por mães que mantiveram sentimentos negativos nos dois momentos, o G2 foi formado por mães que apresentaram sentimentos positivos nos dois momentos e G3 por mães que passaram de sentimentos negativos diante da notícia para sentimentos positivos sobre as visitas a unidade. Esses grupos foram comparados utilizado o teste Anova obtendo resultados estatisticamente significativos de diferença $(p=0,027)$ entre o G1 e G2 nos escores para Ansiedade-Estado, tendo médias maiores no primeiro grupo em que as mães mantiveram sentimentos negativos nos dois momentos. Encontrou-se diferença significativa $(p=0,028)$ entre o G1 e G3 no escore para depressão, sendo o G1 de maior média (Tabela 6). 
Tabela 6 - Comparação da saúde emocional materna dos grupos G1, G2 e G3.

\begin{tabular}{|c|c|c|c|c|c|c|c|c|c|c|c|c|c|c|c|}
\hline & \multicolumn{2}{|c|}{$\mathrm{G} 1(\mathrm{n}=39)$} & \multicolumn{3}{|c|}{$\mathrm{G} 2(\mathrm{n}=11)$} & \multicolumn{2}{|c|}{$\mathrm{G} 1(\mathrm{n}=39)$} & \multicolumn{2}{|c|}{$\mathrm{G} 3(\mathrm{n}=55)$} & \multirow[b]{2}{*}{$\mathrm{p}$} & \multicolumn{2}{|c|}{$\mathrm{G} 2(\mathrm{n}=11)$} & \multicolumn{2}{|c|}{$\mathrm{G} 3(\mathrm{n}=55)$} & \multirow[b]{2}{*}{$\mathrm{p}$} \\
\hline & $\mathrm{M}$ & DP & $\mathrm{M}$ & $\mathrm{DP}$ & $\mathrm{p}$ & $\mathrm{M}$ & DP & M & $\mathrm{DP}$ & & $\mathrm{M}$ & $\mathrm{DP}$ & $\mathrm{M}$ & $\mathrm{DP}$ & \\
\hline Ansiedade-Estado & 49,2 & 8,3 & 42,7 & 8,7 & ,027 & 49,2 & 8,3 & 48,0 & 9,0 & ,488 & 42,7 & 8,7 & 48,0 & 9,0 & ,082 \\
\hline Ansiedade-Traço & 41,7 & 8,3 & 40,6 & 9,3 & ,697 & 41,7 & 8,2 & 42,9 & 9,3 &, 528 & 40,6 & 9,3 & 42,9 & 9,3 & ,455 \\
\hline Estresse & 22,5 & 10,0 & 23,6 & 8,9 &, 739 & 22,5 & 10,0 & 22,2 & 8,7 & 895 & 23,6 & 8,9 & 22,2 & 8,7 & ,635 \\
\hline Depressão & 9,8 & 6,0 & 6,4 & 5,8 & , 101 & 9,8 & 6,0 & 7,2 & 5,3 & ,028 & 6,4 & 5,8 & 7,2 & 5,3 & ,654 \\
\hline $\begin{array}{l}\text { Número de indica- } \\
\text { dores }\end{array}$ & 1,5 & 1,3 & 1,0 & 1,5 &, 345 & 1,5 & 1,3 & 1,3 & 1,2 & ,475 & 1,0 & 1,5 & 1,3 & 1,2 &, 561 \\
\hline
\end{tabular}

Fonte: dados da pesquisa

Legenda: $\mathrm{p}<0,05, \mathrm{DP}=$ Desvio padrão, $\mathrm{M}=$ média

\section{Discussão}

Considerando os sentimentos vivenciados pelas mães observou-se prevalência de diferentes tipos de sentimentos, sendo eles positivos ou negativos como também observado por Pinto et al. (2009) e Hagen, Iversen e Svindseth (2016), a depender da situação. Os sentimentos negativos foram mais frequentes por ocasião da notícia da internação, dado também encontrado por Fonseca (2016). A notícia é acompanhada pela separação abrupta da díade e o pouco contato físico (Jordão et al., 2016), a dúvida iminente sobre a sobrevivência do bebê devido suas condições de risco e as incertezas sobre a condição de saúde do bebê (Zani \& Alvim, 2017). À medida em que havia a habituação ao contexto da UTIN observou-se a redução de sentimentos negativos que foram avaliados durante as visitas da mãe ao bebê internado na UTIN. Neste momento prevaleceram os sentimentos positivos, também relatados por Cordeiro et al. (2018), Fernandes e Silva (2015) e Pinto et al. (2009). Tais sentimentos provavelmente são desencadeados pelo conhecimento dos equipamentos, da rotina da unidade e confiança nos cuidados que estão sendo ofertados pelos profissionais. Fonseca (2016) também atribuiu ao amparo recebido pela rede de apoio presente neste processo. A diferença de sentimentos, prevalência de sentimentos negativos no momento da notícia da internação e sentimentos positivos durante as visitas a unidade também foi relatada por Silva et al. (2016) que sugerem que os primeiros sentimentos estão, principalmente, relacionados a percepção sobre a condição de saúde do bebê e que, após um período de convivência com o ambiente hospitalar, passam a relatar sentimentos positivos.

A avaliação da saúde emocional materna mostrou maior número de mães com indicadores clínicos no constructo Ansiedade-Estado, demonstrando que o contexto da notícia e hospitalização do bebê é um possível desencadeador desses sintomas nas mães, confirmando os dados encontrados por Cruz Dantas et al. (2015). Quanto ao número de indicadores clínico encontrou-se que, 44\% delas apresentaram mais de um indicador clínico para saúde emocional, resultado semelhante ao encontrado por Montanhaur et al. (2021), em que sintomas de ansiedade apareceram correlacionados a sintomas de depressão.

A relação observada entre sentimentos e saúde emocional das mães foi de que aquelas que expressaram sentimentos negativos diante da notícia da internação apresentaram maiores pontuações para Ansiedade-Estado do que as mães que expressaram sentimentos positivos. Ainda que não significativos as mães do G2 também apresentaram médias maiores que as mães com sentimentos positivos em Ansiedade Traço, Depressão e no número de indicadores.

Em relação aos sentimentos expressos durante as visitas obteve-se, também, diferença na saúde emocional das mães que relataram mais sentimentos negativos do que positivos, sendo que o G2 apresentou médias maiores na pontuação para sintomas de depressão do que as mães que tiveram uma percepção positiva do momento. As mães do G2 também apresentaram médias maiores que G1 em Ansiedade Estado, Estresse e no número de indicadores, ainda que sem diferença estatisticamente significativa entre eles. A associação de sentimentos e percepções negativas da internação e visitas a sintomas de ansiedade e depressão também foram encontrados por Correia et al. (2008).

As mães que permaneceram com sentimentos negativos da internação à visita apresentaram pontuações maiores em Ansiedade Estado, Traço, Depressão e maior número de indicadores do que as mães que relataram 
sentimentos positivos durante as duas situações, ainda que somente a diferença em Ansiedade Estado tenha sido significativa. Esse resultado se assemelha ao encontrado por Pinto et al. (2009) que encontraram que percepções positivas foram relacionadas diretamente com baixas pontuações em Ansiedade-Estado. As mães que mudaram para sentimentos positivos durantes as visitas apresentaram pontuações mais baixas do que as que permaneceram com sentimentos negativos em Ansiedade Estado, Estresse, depressão e número de indicadores, com diferença significativa em Depressão. Comparando as mães do G2 (positivos nas duas condições) e do G3 (negativo/positivo) observou-se que as mães do G3 apresentaram maiores pontuações em Ansiedade Traço e Estado, Depressão e número de indicadores do que as mães que no decorrer do processo relataram sentimentos positivos.

\section{Considerações Finais}

O presente projeto pretendeu descrever e comparar sentimento e saúde emocional de mães de bebês internados em UTIN. Os dados mostraram que a fase da internação, especificamente, a ocasião da notícia da mesma, gera sentimentos negativos e altera significativamente a saúde emocional materna, demonstrando ser um momento que deve ser cuidado e acompanhado e que merece cuidados psicológicos dirigidos a mãe no momento do início a alta hospital.

Entre as limitações do estudo houve uma variabilidade no tempo de internação por ocasião da coleta de dados com as mães, uma variável que poderia ser controlada pelo efeito que pode ter sobre sentimentos e saúde emocional. Estudos com populações maiores confirmariam ou refutariam os dados obtidos assim como análises que contemplassem as variáveis sociodemográficas auxiliando na compreensão do fenômeno.

\section{Referências}

Associação Brasileira de Empresas de Pesquisa (ABEP) (2013). Critério de classificação econômica Brasil. São Paulo.

Coelho, A.S., Custódio, D. C. G. G., Rosso, G., Silva, R., Silva, J. S. C., \& Carniel, F. (2018) Equipe de enfermagem e a assistência humanizada na UTI Neonatal. Revista Ciência \& Saberes - FACEMA, Maranhão, 4(1), 873-877. Disponível em: https://www.facema.edu.br/ojs/index.php/ReOnFacema/ article/view/381/176.

Cordeiro, J. S., Cavalcante, L. A., Duarte, A. R. P., Figueira, M. C. S., Jacob, L. M. S., \& Pimenta, C. J. L. (2018) Experiências vivenciadas por mães de recém-nascidos em unidade de terapia intensiva. Revista Intellectus, 1(44), 23-32.

Correia, L. L., Carvalho, A. M. V. \& Linhares, M. B. M. (2008) Conteúdos verbais expressos por mães de bebês prematuros com sintomas emocionais clínicos. Rev. Latino-am. Enfermagem, 16 (1), 64-70. DOI: 10.1590/S010411692008000100011
Cruz Dantas, M.M., Araújo, P.C. B., Revorêdo, L. S., Pereira, H. G., \& Maia, E. M. C. (2015). Mães de recém-nascidos prematuros e a termo hospitalizados: avaliação do apoio social e da sintomatologia ansiogênica. Acta Colombiana de Psicologia, 18(2), 129-138. DOI: 10.14718/ACP.2015.18.2.11

Faro, A. (2015). Análise fatorial confirmatória das três versões da Perceived Stress Scale (PSS): Um estudo populacional. Psicologia, Reflexão e Crítica, 28(1), 21-30. DOI: 10.1590/1678-7153.201528103

Fonseca, M.C.S. (2016) Humanização na relação mãe/pai/ bebê prematuro em uma UTI Neonatal: a separação precoce. 2016. Dissertação (Mestrado em Família na Sociedade Contemporânea) - Pró-Reitoria de Pesquisa e Pós-Graduação, Universidade Católica do Salvador, Salvador.

Hagen, I.H., Iversen, V.C., \& Svindseth, M.F. (2016). Differences and similarities between mothers and fathers of premature children: a qualitative study of parents' coping experiences in a neonatal intensive care unit. $B M C$ Pediatrics, 16(1), 1-5. Disponível em: https://bmcpediatr.biomedcentral. com/articles/10.1186/s12887-016-0631-9. Acesso: 24 jul. 2019.

Jordão, K. R., Pinto, L. A. P., Machado, L. R., Costa, L. B. V. L. \& Trajano, E. T. L. (2016). Possíveis fatores estressantes na unidade de terapia intensiva neonatal em hospital universitário. Rev. Bras. Ter. Intensiva, 28(3), 310-314. DOI:10.5935/0103-507X.20160041

Margaret, B., Lewis, L. E., Bhat, Y. R., Nayak, B. S., Pai, M. S. \& Mundkur, S. C. (2018). Impact of Depressive Symptoms on Mother Infant Attachment among Mothers of Preterm Neonates. Online Journal of Health and Allied Sciences, 17(1), 1-5. Acesso em 24 de junho de 2019. Disponível em: https://www.doaj.org/article/10ba0045454a4c 1bb04144fc0c4d7b62.

Melo, R. A., Araújo, A. K. C., Bezerra, C. S., Santos, N. M., Marques, W. F. \& Fernandes, F. E. C. V. (2016). Sentimentos de mães de recém-nascidos internados em uma unidade de terapia intensiva neonatal. Id on Line Revista Multidisciplinar e de Psicologia, 10(32), 88-103. Disponível em: https:// idonline.emnuvens.com.br/id/article/ view/569/786

Montanhaur, C.D. Percepção de mães de bebês internados em uti neonatal: influência de variáveis maternas, contextuais, apoio social e enfrentamento. Dissertação de Mestrado. Programa de Pós-graduação em Psicologia do Desenvolvimento e Aprendizagem. Faculdade de Ciências. UNESP, Bauru, 2018.

Montanhaur, C. D., Rodrigues, O. M. P. R., \& Arenales, N. G. (2021). Saúde emocional materna e tempo de internação de neonatos. Aletheia, 54(1), 55-63. DOI: $10.29327 / 226091.54 .1-6$

Neves, R. S., Zimmermann, J., \& Broering, C. V. (2021) UTI Neonatal: o que dizem as mães. Psicologia e Saúde em Debate, 7(1), 187-214. DOI: 10.22289/2446-922X.V7N1A14

World Health Organization (WHO). Survive and Tbrive - Transforming care for every small and sick newborn. Genebra, 2018.

Pinto, I. D., Padovani, F. H. P., \& Linhares, M. B. M. (2009). Ansiedade e depressão materna e relatos sobre o bebê prematuro. Psicologia: Teoria e Pesquisa, 25(1), 75-83. DOI:10.1590/S0102-37722009000100009

Roseiro, C.P., \& Paula, K.M.P. (2015). Concepções de humanização de profissionais em Unidades de Terapia Intensiva Neonatal. Estudos de Psicologia, Campinas, 32(1), 109-119. DOI:10.1590/0103-166X2015000100010

Schiavo, R. A., Rodrigues, O. M. P. R., \& Perosa, G. B. (2014). Variáveis associadas à ansiedade gestacional em primigestas e multigestas. Trends in Psychology, 26 (4), 2091-2104. DOI: 10.9788/TP2018.4-14Pt

Silva, R. M. M., Menezes, C. C. S., Cardoso, L. L. \& França, A. F. O. (2016). Vivências de famílias de neonatos prematuros hospitalizados em unidade de terapia intensiva neonatal: revisão integrativa. Enferm. Cent. O. Min, 6(2), 2258-2270. DOI:10.19175

Veronez, M. et. al. (2017). Vivências de mães de bebês prematuros do nascimento a alta: notas de diários de campo. Revista Gaúcha de Enfermagem, Porto Alegre, 38(2),1-8. Disponível em: http://www.scielo.br/scielo. php?pid $=$ S1983-14472017000200419\&script $=$ sci_abstract

Zani, A. V., \& Alvim, H. C. (2017). O filho prematuro de baixo peso: a maternagem hospitalizada. Rev Enferm UFPE on line., Recife, 11(4), 1724-30. DOI: 10.5205/reuol.10438-93070-1-RV.1104sup201715

Submetido em: 8-10-2021

Aceito em: 13-10-2021 